\title{
Original Article \\ Bioaccumulation of Polycyclic Aromatic Hydrocarbons (PAHs) in Green Mussels (Perna viridis) from Cangio Area, Hochiminh city
}

\author{
Hoang Thi Thanh Thuy ${ }^{1, *}$, Pham Thanh Luu ${ }^{2}$, Tu Thi Cam Loan ${ }^{1}$, \\ Nguyen Van Dong ${ }^{3}$, Le Duy Bao ${ }^{3}$, Tran Thi Hoang Yen², Do Xuan Huy ${ }^{4}$ \\ ${ }^{1}$ Ho Chi Minh City University of Natural Resources and Environment, \\ 236B Le Van Sy Street, Hochiminh city, Vietnam \\ ${ }^{2}$ Institute of Tropical Biology, VAST, 85 Tran Quoc Toan Street, Hochiminh city, Vietnam \\ ${ }^{3}$ University of Science Hochiminh city, 227 Nguyen Van Cu Street, Hochiminh city, Vietnam \\ ${ }^{4}$ Vietnam Institute for Environment and Resources, 142 To Hien Thanh Street, Hochiminh city, Vietnam \\ Received 30 January 2020 \\ Revised 05 March 2020; Accepted 09 March 2020

\begin{abstract}
The bioaccumulation of polycyclic aromatic hydrocarbons (PAHs) in bivalves of the coastal estuary has been investigated since they are favorable seafood. In the present study, the distribution of PAHs was determined in the green mussels (Perna viridis) from Cangio area (Hochiminh city). Global comparison showed a lower PAHs accumulated in the green mussels, which ranged between 2.97 to $15.79 \mathrm{ng} / \mathrm{g}$ wet weight (ww). However, the presence of carcinogenic isomer (benzo[ $[a]$ pyrene) (up to $0.15 \mathrm{ng} / \mathrm{g} \mathrm{ww}$ ) emphasizes a further monitoring program to ensure the food quality and community health.
\end{abstract}

Keywords: Polycyclic aromatic hydrocarbons, bioaccumulation, green mussels, Cangio.

\footnotetext{
* Corresponding author.

E-mail address: thuyhoang.geo@gmail.com

https://doi.org/10.25073/2588-1094/vnuees.4551
} 


\title{
Tích lũy hydrocarbon thơm đa vòng (PAHs) trong vẹm xanh (Perna viridis) ở khu vực Cần Giờ, Thành phố Hồ Chí Minh
}

\author{
Hoàng Thị Thanh Thủy ${ }^{1, *}$, Phạm Thanh Lưư ${ }^{2}$, Từ Thị Cẩm Loan ${ }^{1}$, \\ Nguyễn Văn Đông ${ }^{3}$, Lê Duy Bảo ${ }^{3}$, Trần Thị Hoàng Yến ${ }^{2}$, Đỗ Xuân Huy ${ }^{4}$ \\ ${ }^{I}$ Truoòng Đại học Tài nguyên và Môi truờng TP. HCM, 236B Lê Văn Sỹ, TP. HCM, Việt Nam \\ ${ }^{2}$ Viện Sinh học nhiệt đới, VAST, 85 Trần Quốc Toản, TP. HCM, Việt Nam \\ ${ }^{3}$ Truòng Đại học Khoa học tư nhiên TP.HCM, 237 Nguyễn Văn Cù̀, TP. HCM, Việt Nam \\ ${ }^{4}$ Viện Môi truờng và Tài nguyên, 142 Tô Hiến Thành, TP. HCM, Việt Nam \\ Nhận ngày 30 tháng 01 năm 2020 \\ Chỉnh sửa ngày 05 tháng 3 năm 2020; Chấp nhận đăng ngày 09 tháng 3 năm 2020
}

\begin{abstract}
Tóm tắt: Sự tích lũy của Hydrocacbon thơm đa vòng (PAHs) trong các sinh vật hai mảnh vỏ ở các khu vực cửa sông ven biển đã được rất nhiều nhà khoa học trên thế giới quan tâm vì các hải sản luôn là một thực phẩm được ưa chuộng. Bài báo này trình bày kết quả nghiên cứu ban đầu về hàm lượng PAHs tích lũy trong một sinh vật hai mảnh vỏ điển hình là Vẹm xanh (Perna viridis) ở khu vực Cần Giờ (Thành phố Hồ Chí Minh). Kết quả nghiên cứu đã cho thấy ở khu vực Cần Giờ hàm lượng PAHs trong Vẹm xanh còn khá thấp (biến thiên từ 2,97 đến $15,79 \mathrm{ng} / \mathrm{g}$ trọng lượng ướt-ww) so với nhiều khu vực khác trên thế giới. Tuy nhiên, đã có sự xuất hiện của đồng phân có khả năng gây ung thư (benzo[ $[a]$ pyrene) (hàm lượng cao nhất là $0,15 \mathrm{ng} / \mathrm{g}$ ww) nên rất cần triển khai các chương trình quan trắc thường xuyên đảm bảo chất lượng thực phẩm và sức khỏe cộng đồng.
\end{abstract}

Tù khóa: Hydrocacbon thơm đa vòng, tích lũy sinh học, Vẹm xanh, Cần Giờ.

\section{Mở đầu}

Các hydrocacbon thơm đa vòng (Polycyclic Aromatic Hydrocarbon - $\mathrm{PAHs}$ ) là các hợp chất hữu cơ chỉ chứa $\mathrm{C}$ và $\mathrm{H}$, có hai hay nhiều vòng thơm. Trong môi trường, có hai nguồn phát thải PAHs là từ quá trình đốt cháy (cháy rừng, đốt nhiên liệu,...) và từ xăng dầu (tràn dầu, tháo lắp

\footnotetext{
* Tác giả liên hệ.

Địa chỉ email: thuyhoang.geo@gmail.com
}

https://doi.org/10.25073/2588-1094/vnuees.4551 vật liệu,...) [1]. Nhóm hợp chất ô nhiễm này đã được nghiên cứu rộng rãi trên thế giới từ những năm 80 của thế kỉ XX do có khả năng gây ung thư và đột biến cho con người [2]. PAHs là nhóm hợp chất khó phân hủy trong điều kiện tự nhiên do đó có khả năng di chuyển và tích lũy trong chuỗi sinh học. Các loại hải sản được ưa chuộng sử dụng làm thực phẩm như sinh vật hai mảnh 
vỏ (hàu, nghêu, vẹm,...) do có tập tính ăn lọc nên khả năng tích lũy các chất ô nhiễm, kể cả PAHs, tương đối cao. Nhiều nghiên cứu trên thế giới đã ghi nhận PAHs tích lũy nhiều ở các động vật thân mềm như nghêu, hàu, sò, vẹm, ốc đặc biệt là ở các vùng cảng, cửa sông ven biển nơi có nhiều hoạt động giao thông thủy. Ở Việt Nam, khi khảo sát hàm lượng PAHs tích lũy trong một số sinh vật biển gồm sò gạo (Anadara subcreanata), ngao trắng (Meretrix lyrata) và ngao vàng (Meretrix meretrix) ở vùng ven bờ Hải Phòng, Phạm Thị Kha (2014) [1] ghi nhận hàm lượng tổng PAHs trong các loài nhuyễn thể hai mảnh vỏ từ $56,41-246,39 \mathrm{ng} / \mathrm{g} \mathrm{dw}$, trong tôm từ 32,48 $-385,46 \mathrm{ng} / \mathrm{g} \mathrm{dw}$ và trong loài cá từ $34,47-$ $517,05 \mathrm{ng} / \mathrm{g} \mathrm{dw}$. Trong các mẫu sinh vật, các PAHs 4-5 vòng chiếm chủ yếu từ 45,85-100\%. Chính vì vậy, sự tích lũy của PAHs trong sinh vật hai mảnh vỏ đã được rất nhiều nhà khoa học trong và ngoài nước quan tâm để đảm bảo chất lượng thực phẩm.

Cần Giờ là huyện ven biển nằm phía Đông Nam thuộc Thành phố Hồ Chí Minh (TP. HCM), có bờ biển dài $20 \mathrm{~km}$ chạy dọc theo hướng Đông Nam - Tây Bắc. Cần Giờ không những là nơi khai thác thủy hải sản với sản lượng đáng kể mà còn là nơi có điều kiện thuận lợi để nuôi trồng nhiều loài hải sản có giá trị kinh tế cao. Hiện tại, các nghiên cứu về tích lũy PAHs trong các sinh vật hai mảnh vỏ ở khu vực này còn khá hạn chế. Bài báo này trình bày kết quả nghiên cứu ban đầu về hàm lượng PAHs tích lũy trong sinh vật hai mảnh vỏ điển hình là Vẹm xanh (Perna viridis) và bước đầu đánh giá rủi ro đến sức khỏe con người khi sử dụng sinh vật này làm thực phẩm.

\section{Phương pháp nghiên cứu}

\subsection{Phuoong pháp thu mẫu}

Mẫu Vẹm xanh $P$. viridis được thu ở 4 vị trí có kí hiệu lần lượt là $\mathrm{CG} 1, \mathrm{CG} 2, \mathrm{CG} 3$ và $\mathrm{CG} 4$ tại vùng cửa biển Cần Giờ, TP. HCM. Hai đợt thu mẫu đã được triển khai là tháng 9 năm 2017 (mùa mưa) và tháng 4 năm 2018 (mùa khô). Tại mỗi vị trí thu từ 5 đến 10 cá thể, mẫu sau khi thu sẽ được rửa sạch bùn tại hiện trường và giữ lạnh ở $4^{\circ} \mathrm{C}$ cho đến khi về phòng thí nghiệm. Trong phòng thí nghiệm mẫu được rửa sạch bằng nước cất, sau đó đo kích thước vỏ, cân trọng lượng tươi của mỗi cá thể. Mẫu được loại bỏ phần vỏ cứng, thu giữ phần mô mềm và lưu trữ ở $-20^{\circ} \mathrm{C}$ cho đến khi phân tích (Hình 1).

\subsection{Phurong pháp tách chiết và phân tích PAHS}

Phương pháp tách chiết PAHs trong mẫu Vẹm xanh được thực hiện theo Fang và cộng sự (2009) cùng một số cải tiến [3]. Theo đó, bốn gram mẫu mô đã được đồng nhất, loại lượng chất béo có trong mẫu bằng cách lắc với hỗn hợp 10 $\mathrm{mL} \mathrm{HCl}$ đậm đặc và $20 \mathrm{~mL}$ chloroform trong 12 giờ ở tốc độ $200 \mathrm{rpm}$. Tiếp theo, mẫu được ly tâm ở $3.000 \mathrm{rpm}$ trong 5 phút, loại acid thừa bằng $2 \times 10 \mathrm{~mL}$ nước khử ion. Thu pha hữu cơ và loại nước (nếu có) bằng $\mathrm{Na}_{2} \mathrm{SO}_{4}$ khan, thêm $2 \mathrm{~mL}$ iso octan. Sau đó cô quay mẫu còn khoảng $1 \mathrm{~mL}$ trong điều kiện nhiệt độ: $40^{\circ} \mathrm{C}$, vận tốc: $40 \mathrm{rpm}$, áp suất: 600 mbar. Mẫu sau cô quay được tách chiết PAHs bằng cột chiết qua pha rắn $(4 \mathrm{~g}$ silicagel, $4 \mathrm{~g}$ alumina đã được giảm hoạt $5 \%$ ). Trình tự tách chiết được thực hiện qua 2 giai đoạn i) Làm sạch cột bằng $5 \times 2 \mathrm{~mL}$ n-pentan và ii) Rửa giải PAHs với $3 \times 20 \mathrm{~mL}$ hỗn hợp dung môi n-pentane: dichloromethane (80:20, v/v).

Bổ sung $1 \mathrm{~mL}$ DMSO vào dịch chiết thu được, sau đó cô quay đến còn khoảng $1 \mathrm{~mL}$ trong điều kiện nhiệt độ: $25^{\circ} \mathrm{C}$, vận tốc: 40rpm, áp suất: 450 mbar. Chuyển và tráng mẫu sau cô quay bằng acetonitrile vào vial dung tích $1,5 \mathrm{~mL}$.

Mười lăm đồng phân của PAHs (Naphthalene (Nap), Acenaphthene (Ace), fluorene (Fl), phenanthrene (Phe), anthracene (Ant), fluoranthene (Flu), pyrene (Pyr), benzo[ $a]$ anthracene $(\mathrm{BaA})$, chrysene $(\mathrm{Ch})$, benzo[b]fluoranthene $(\mathrm{BbF})$, benzo[ $k]$ fluoranthene $(\mathrm{BkF})$, benzo[ $a]$ pyrene $(\mathrm{BaP}), \quad$ dibenzo[ $[a, h]$ anthracene $(\mathrm{dBA})$, benzo[ $g, h, i]$ perylene $(\mathrm{BgP})$ và indeno[1,2,3cd]pyrene $(\mathrm{InP})$ ] được phân tích trên thiết bị HPLC-FLD (Dionex UltiMate 3000, Thermo Scientific, Waltham, MA USA) bằng cột $\mathrm{C}_{8}$ (cột PAH Eclipse $3,0 \times 250 \mathrm{~mm}, 5 \mu \mathrm{m}$, Agilent) và tiền cột persuit PAH (AcclaimM $120 \mathrm{C}_{18} 5 \mu \mathrm{m}$, $4.6 \times 150 \mathrm{~mm}$, Waltham, MA USA, Ireland). 


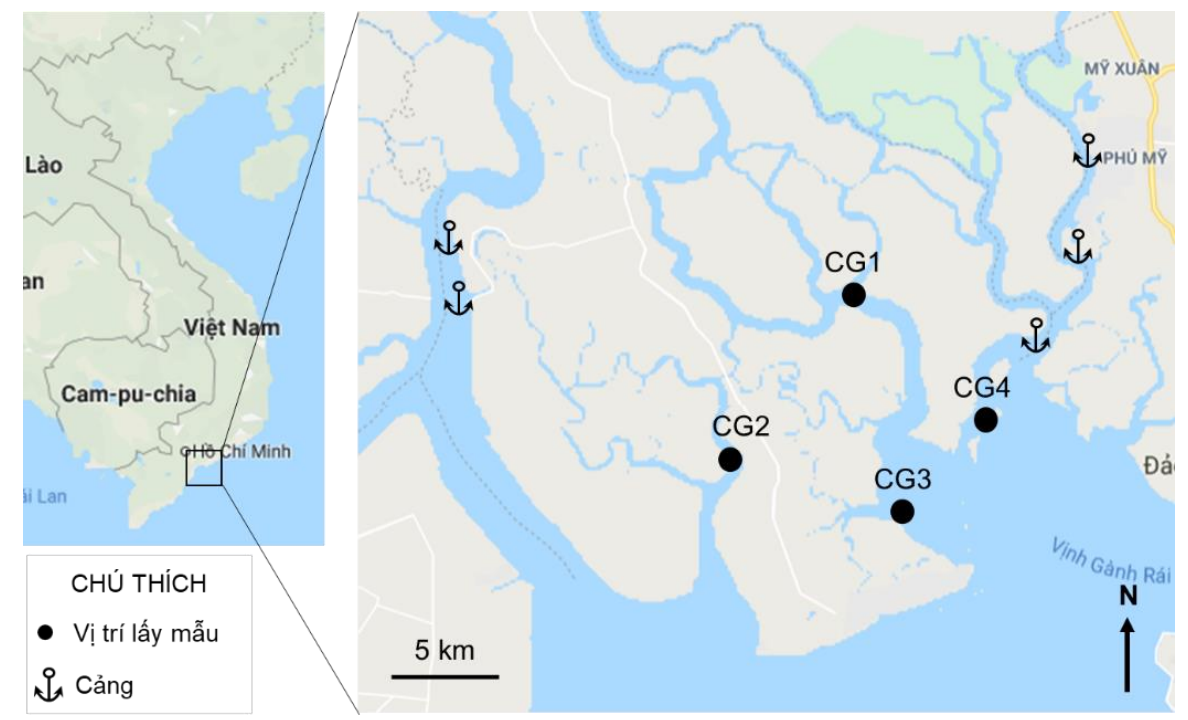

Hình 1. Sơ đồ vị trí thu Vẹm xanh (Perna viridis) tại khu vực Cần Giờ, TP.HCM.

Bảng 1. Giới hạn phát hiện của phương pháp (MDL) và độ thu hồi (\%) PAHs

\begin{tabular}{cccccccccccccccc}
\hline PAHs & Nap & Acc & Flu & Phe & Ant & Flt & Pyr & BaA & Chr & BbF & BkF & BaP & dBA & BgP & InP \\
\hline MDL (ng/g) & 0,67 & 0,51 & 0,22 & 0,24 & 0,09 & 0,18 & 0,2 & 0,23 & 0,2 & 0,14 & 0,14 & 0,12 & 0,38 & 1,4 & 3,6 \\
Đô thu hồi (\%) & 112 & 81 & 79 & 103 & 82 & 87 & 102 & 94 & 97 & 93 & 84 & 72 & 81 & 79 & 82 \\
\hline
\end{tabular}

Quy trình kiểm soát chất lượng được thực hiện bằng các mẫu chuẩn và mẫu lặp cho từng đợt đo (10 mẫu). Giới hạn phát hiện của phương pháp (Method detection limit - MDL) thấp nhất là $0,09 \mathrm{ng} / \mathrm{g}(\mathrm{Ant})$ và cao nhất là $3,6 \mathrm{ng} / \mathrm{g}$ (Inp) (Bảng 1). Độ thu hồi nằm trong khoảng $72 \%$ (BaP) và $112 \%$ (Nap). Sai số giữa hai mẫu lặp thấp hơn $15 \%$.

\section{3. Đánh giá rủi ro và sức khỏe con ngườ}

Nguy cơ sức khỏe con người liên quan đến việc tiêu thụ Vẹm xanh được ước tính theo các phương pháp của Zhao và cộng sự (2014) [4].

Chỉ số nồng độ có thề có ảnh hưởng (Probable effect concentration - PEC) của PAHs được tính theo công thức sau (1):

$$
P E C=\sum_{i=1}^{n} T E F_{i} \times C_{i}
$$

Trong đó:

TEF: các yếu tố gây độc tương đương $\mathrm{BaP}$ của từng đồng phân $\mathrm{PAH}$ riêng lẻ.

$\mathrm{C}_{\mathrm{i}}$ : nồng độ của mỗi $\mathrm{PAH}$ được phát hiện trong mẫu i.
Theo US EPA, 2000, giá trị TEF được xác định như sau: 0,001 (Nap, Acc, Flu, Flt, Phe và Pyr); 0,01 (Ant, Chr và $\mathrm{BgP}) ; 0,1(\mathrm{BaA}, \mathrm{BbF}$, $B k F$ và $I n P)$ và 1 cho $B a P$ và $d B A$.

Thêm vào đó, chỉ số rủi ro ung thư (Excess cancer risk - ECR) được sử dụng để tính toán mức độ rủi ro ung thư suốt đời khi sử dụng Vẹm xanh là thức ăn theo phương pháp của Ke và cộng sự, 2017 [5] như sau:

$$
E C R=\frac{C S F \times P E C \times C R \times E D}{B W \times A T} \times 10^{-6}
$$

Trong đó:

CSF: hệ số ung thư qua đường miệng của $\mathrm{BaP}$ là $7,3 \mathrm{mg} / \mathrm{kg} /$ ngày [5].

CR: mức tiêu thụ hải sản trung bình cho các nước châu Á ở khu vực thành thị là $7 \mathrm{~g} /$ người/ ngày [6]. năm).

ED: thời gian phơi nhiễm (người lớn $=30$

BW: trọng lượng trung bình của người trưởng thành châu Á là $60 \mathrm{~kg}$.

AT: tuổi thọ trung bình (70 năm) [7]. 


\subsection{Phưong pháp xủ lý số liệu}

Số liệu về hàm lượng PAHs tích lũy trong Vẹm xanh được phân tích thống kê bằng phần mềm SPSS 20.0 và Excel 2016.

\section{Kết quả và thảo luận}

\subsection{Hàm lựng PAHs tích lũy trong vẹm xanh}

Kết quả cho thấy hàm lượng PAHs tích lũy trong Vẹm xanh vẫn ở mức thấp. Chỉ có 6 đồng phân PAHs (Nap, Phe, Flt, Chr, BbF và $\mathrm{BaP}$ ) được phát hiện trong tất cả các mẫu với tổng hàm lượng ( $\Sigma \mathrm{PAHs}$ ) từ 2,97-15,79 ng/g trọng lượng ướt (ww). Các đồng phân Acc, Flu, Ant, Pyr, $\mathrm{BaA}, \mathrm{BkF}, \mathrm{dBA}, \mathrm{BgP}$ và $\mathrm{InP}$ đều nhỏ hơn giới hạn phát hiện (Bảng 2). Nhìn chung, hàm lượng PAHs trong Vẹm xanh ở khu vực Cần giờ khá thấp so với các nghiên cứu trước đây ở Malaysia $(12-1.690 \mathrm{ng} / \mathrm{g}$ ww) và Cuba $(47,38-89,30 \mathrm{ng} / \mathrm{g}$ ww) $[8,9]$.

Bảng 2. Nồng độ PAH (ng/g ww) trong mô của Vẹm xanh (Perna viridis sp.)

\begin{tabular}{lllll}
\hline \multirow{2}{*}{ PAHs } & \multicolumn{3}{l}{ Tháng 9/2017 } & \multicolumn{2}{l}{ Tháng 4/2018 } \\
\cline { 2 - 5 } & Min - Max & Std & Min - Max & Std \\
\hline Nap & $1,49-3,03$ & 0,59 & $0,87-2,90$ & 0,79 \\
Acc & KPH & - & KPH & - \\
Flu & KPH & - & KPH & - \\
Phe & $1,26-4,37$ & 1,31 & $0,77-1,75$ & 0,33 \\
Ant & KPH & - & KPH & - \\
Flt & $0,69-2,13$ & 0,56 & 1,03 & 0,37 \\
Pyr & KPH & - & KPH & - \\
BaA & KPH & - & KPH & - \\
Chr & 1,13 & 0,50 & KPH & - \\
BbF & $0,21-1,96$ & 0,65 & $1,33-3,50$ & 0,79 \\
BkF & KPH & - & KPH & - \\
BaP & 3,17 & 1,19 & 0,37 & 0,15 \\
dBA & KPH & - & KPH & - \\
BgP & KPH & - & KPH & - \\
InP & KPH & - & KPH & - \\
LPAHs & $3,65-15,79$ & - & $2,97-9,55$ & - \\
\hline
\end{tabular}

Ghi chú: Min: Giá trị nhỏ nhất, Max: Giá trị lớn nhất, Std: độ lệch chuẩn, ww: Trọng luợng uớt; KPH: không phát hiện

\subsection{Sụ thay đổi theo thời gian và không gian của PAHs trong Vem xanh}

Giữa hai mùa không có sự khác biệt có ý nghĩa thống kê đối với $\Sigma$ PAHs trong Vẹm xanh (Sig. $=0,77)$. Tuy nhiên, vào mùa mưa (tháng 9 năm 2017) có xu thế cao hơn so với mùa khô (47 ng/g ww so với 37 ng/g ww) (Hình 2A). Xu thế tăng cao vào mùa mưa có thể phản ánh sự vận chuyển các chất ô nhiễm này do dòng chảy bề mặt vào khu vực Cần Giờ.

Hàm lượng $\mathrm{SPAHs}$ được phát hiện trong Vẹm xanh cũng không thể hiện sự khác biệt có ý nghĩa thống kê giữa các vị trí trong đất liền (CG1 và $C G 2)$ và ven biển $(C G 3$ và $C G 4)(S i g .=0,78$ và 0,18 cho hai đợt lấy mẫu). Nhưng nhìn chung các vị trí bên trong đất liền đã thể hiện xu thế tăng cao hơn so với ngoài cửa sông (Hình 2B). Như đã trình bày ở trên, PAHs có thể phát sinh từ các hoạt động trong đất liền, được vận chuyển bằng dòng chảy bề mặt và sau đó khi ra đến vùng cửa sông thì bị pha loãng.

\subsection{Sự phân bố của các đồng phân PAHs trong Vẹm xanh}

Thành phần của các PAHs trong Vẹm xanh (Perna viridis) ở khu vực Cần Giờ được trình bày ở Hình 3. Các đồng phân có cấu trúc 2 - 3 vòng thơm chiếm ưu thế so với các đồng phân có số vòng cao hơn. Mức độ phổ biến của các đồng phân được sắp xếp như sau:

$\mathrm{PAH}_{2-3}$ vòng $(40-50 \%)>\mathrm{PAH}_{5-6}$ vòng $(33-$ $40 \%)>\mathrm{PAH}_{4 \text { vòng }}(17-20 \%)$

Kết quả này phù hợp với các nghiên cứu trước đây trên thế giới. Nghiên cứu trước đây của Yu và cộng sự (2016) [10] cũng đã nhấn mạnh rằng động vật hai mảnh vỏ có xu hướng tích lũy đến $80 \%$ các PAHs có trọng lượng phân tử thấp. Tương tự, tỷ lệ trên $80 \%$ các $\mathrm{PAHs}$ có trọng lượng phân tử thấp cũng đã được nêu trong các nghiên cứu của Shahbazi \& cộng sự (2010), Miguel - Gallo \& cộng sự (2017) [8,9]. Tuy nhiên, sự có mặt của các PAHs có trọng lượng phân tử cao (có năm đến sáu vòng thơm) cũng cần được nghiên cứu chi tiết hơn vì các $\mathrm{PAHs}$ này rất bền trong điều kiện tự nhiên nên có thể ảnh hưởng đến sức khỏe người khi sử dụng Vẹm xanh là thực phẩm. 

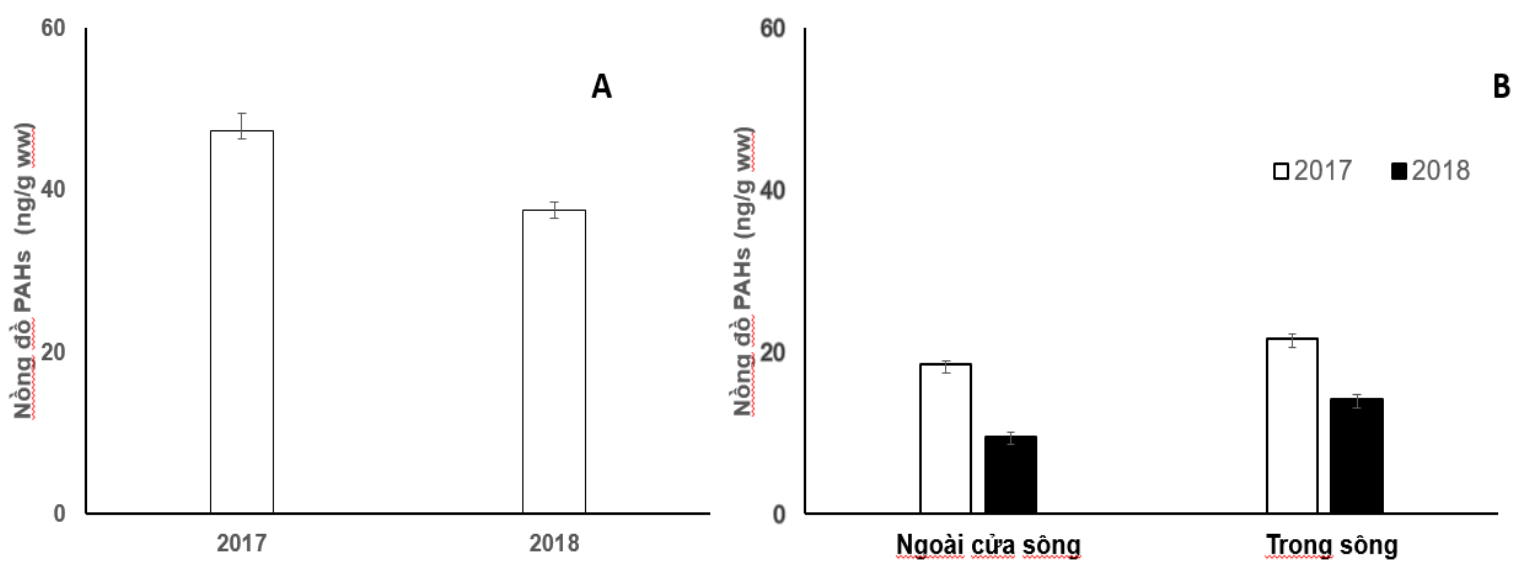

Hình 2. Sự biến đổi theo thời gian (A) và không gian (B) của $\Sigma$ PAHs tích lũy trong Vẹm xanh Perna viridis.

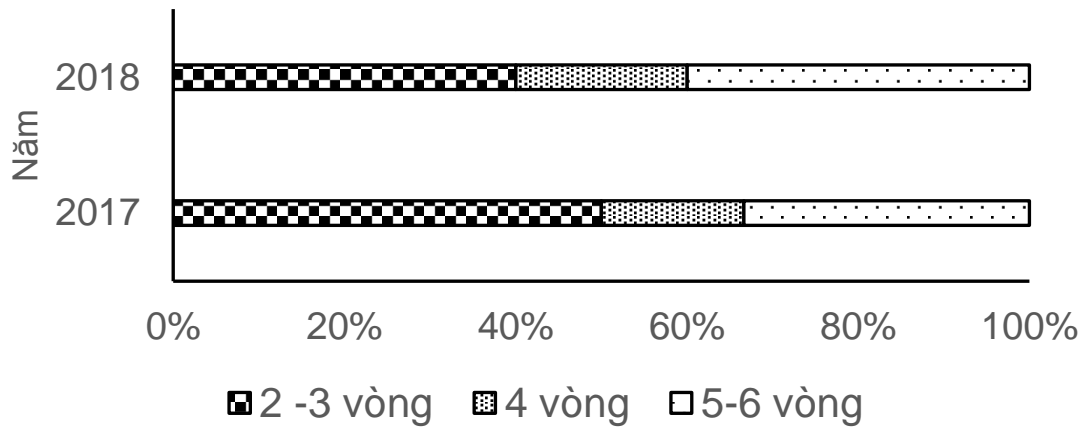

Hình 3. Thành phần của PAHs có trong Vẹm Perna viridis.
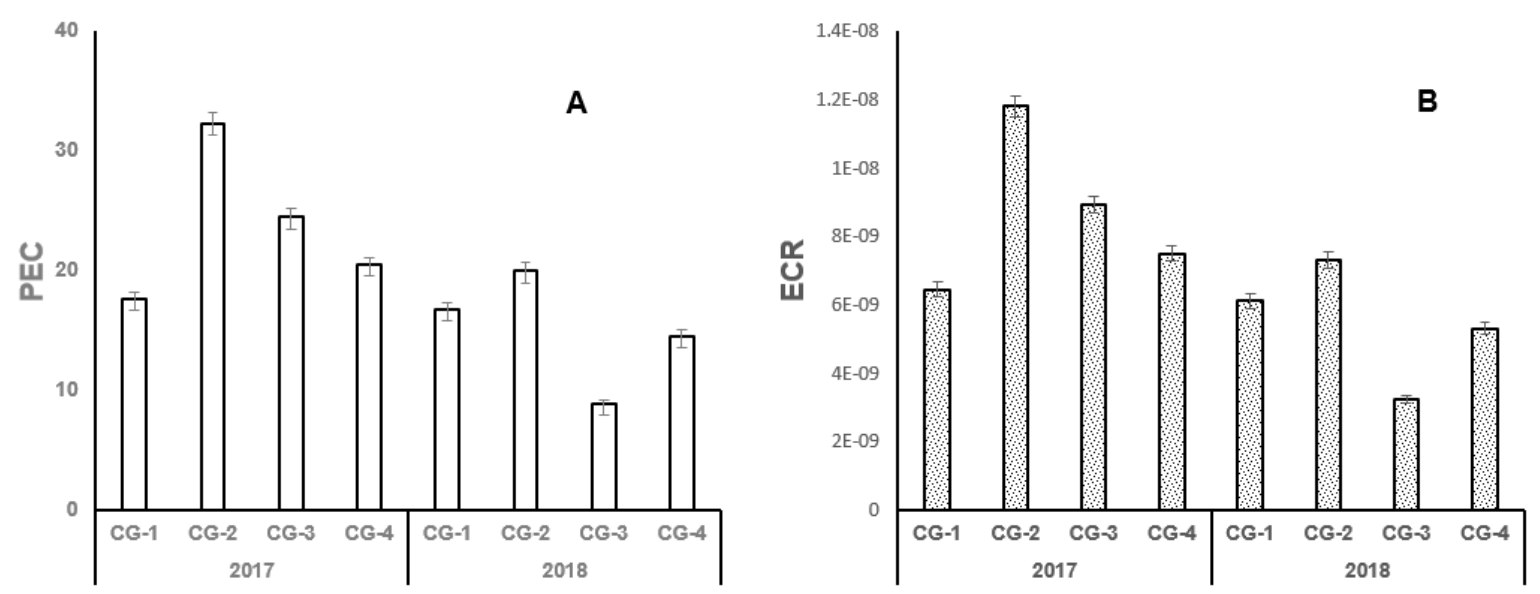

Hình 4. Nồng độ tương đương tiềm năng (PEC) (A) và nguy cơ ung thư vượt mức (ECR) (B) có trong Vẹm (Perna viridis) tại khu vực Cần Giờ. 


\section{4. Đánh giá rủi ro súc khỏe con người}

Khi PAHs tích lũy trong sinh vật được sử dụng là thực phẩm thì có thể gây nguy cơ ung thư cho con người. Do đó, nhiều quốc gia và tổ chức đã xác định giới hạn đối với PAHs trong sinh vật, đặc biệt đối với BaP là đồng phân PAHs có khả năng gây ung thư $[4,5,11]$. Chính vì vậy, giá trị giới hạn của PAHs trong thực phẩm đã được đề xuất bởi Công ước Olso và Paris (OSPAR) ở Châu Âu, Cục Quản lý Thực phẩm và Dược phẩm (FDA) và Ủy ban Châu Âu (EC) [12]. Ở Việt Nam chưa có quy định cụ thể về PAHs trong sinh vật làm thực phẩm. Do đó, để đánh giá rủi ro đến sức khỏe cộng đồng, tiêu chuẩn EC No 1881/2006 của cộng đồng Châu Âu đã được áp dụng. Theo tiêu chuẩn này giá trị giới hạn của $\mathrm{BaP}$ đối với nhuyễn thể hai mảnh vỏ là $10 \mathrm{ng} / \mathrm{g}$.

Ở khu vực Cần Giờ, BaP được phát hiện trong Vẹm xanh ở cả hai mùa, với xu thế tăng trong mùa mưa 2017 so với mùa khô năm 2018 (3,17 và $0,37 \mathrm{ng} / \mathrm{g}$ ww). Hàm lượng $\mathrm{BaP}$ trong Vẹm xanh khu vực Cần Giờ vẫn còn ở mức thấp và đạt yêu cầu của tiêu chuẩn EC No 1881/2006.

Một cách khác, chỉ số PEC đã được xác định là một công cụ hiệu quả để đánh giá độc tính tiềm năng so với tổng nồng độ PAHs $[7,8]$. Ở khu vực Cần Giờ, giá trị PEC của $\Sigma \mathrm{PAHs}$ trong Vẹm xanh thay đổi tương ứng từ 17,67 đến $32,35 \mathrm{ng} / \mathrm{g}$ ww vào tháng $9 / 2017$ và từ 8,89 đến 20,01 vào tháng 4/2018 (Hình 4A).

Giá trị này khá thấp so với nghiên cứu trước đây ở cá từ Trung Quốc (79,46-1.334) [11]. Bên cạnh đó, giá trị $\mathrm{ECR}$ nằm trong khoảng từ $0,32 \times$ $10^{-9}$ dến $1,12 \times 10^{-8}$ (giá trị trung bình $0,58 \times 10^{-8}$ ), thấp hơn nhiều so với giá trị quan sát được trong hải sản từ các quốc gia khác như Trung Quốc $\left(1,2 \times 10^{-5}\right)$, Hàn Quốc $\left(2,35 \times 10^{-5}\right)$ và Tây Ban Nha $\left(4,4 \times 10^{-5}\right)[11,13,14]$. Đồng thời, theo Shi và cộng sự (2016) [11], ECR có giá trị $\left(10^{-6}\right)$ trong vòng đời $(70$ năm) được coi là nồng độ chấp nhận được, trong khi ECR có giá trị $\left(10^{-4}\right)$ được coi là một mức độ rủi ro nghiêm trọng.

Giá trị ECR trong Vẹm từ khu vực Cần Giò̀ thấp hơn đáng kể so với mức chấp nhận được, cho thấy hiện tại chưa có nguy cơ gây ung thư khi sử dụng Vẹm xanh làm thực phẩm. Tuy nhiên, do các mẫu Vẹm xanh được thu chủ yếu tại các bè nuôi hàu nên PAHs cũng đã có thể tích lũy trong các sinh vật hai mảnh vỏ này. Do đó, việc quan trắc hiện trạng $\mathrm{PAHs}$ tích lũy trong sinh vật hai mảnh vỏ ở khu vực Cần Giờ là cần thiết.

\section{Kết luận}

Kết quả nghiên cứu cho thấy có sự tích lũy PAHs trong Vẹm xanh tại khu vực Cần Giờ. Nguồn gốc của PAHs có thể do các hoạt động trong đất liền được vận chuyển ra vùng cửa sông ven biển. Hàm lượng PAHs tích lũy trong Vẹm xanh còn ở mức thấp và chưa thể hiện nguy cơ gây rủi ro sức khỏe. Tuy nhiên, đã sự xuất hiện của đồng phân có khả năng gây ung thư $(\mathrm{BaP})$. Do đó, cần có các chương trình quan trắc thường xuyên để đảm bảo chất lượng nguồn thực phẩm và bảo vệ sức khỏe cộng đồng.

\section{Lò̀i cảm ơn}

Nghiên cứu này được tài trợ bởi Quỹ Phát triển khoa học và công nghệ Quốc gia (NAFOSTED) trong đề tài mã số 105.08 2016.06.

\section{Tài liệu tham khảo}

[1] P.T. Kha, Risk of Polycyclic Aromatic Hydrocarbons (PAHs) accumulation in selected marine organisms in coastal area of Haiphong-Quangninh provinces. Vietnam Journal of Marine Science and Technology 14 (2014) 97-102. (in Vietnamese).

[2] S. Abdelghani, C. Abdelghani, T. Mohamed and H.Budzinski, Origin and Distribution of Polycyclic Aromatic Hydrocarbons in Lagoon Ecosystems of Morocco - The Open Environmental Pollution \& Toxicology Journal 3 (2012) 37-46. https://doi.org/ 10.2174/1876397901203010037.

[3] J.K.H. Fang, D.W.T. Au, R.S.S. Wu, G.J. Zheng, A.K.Y.Chan, P.K.S.Lam and P.K.S. Shin, Concentrations of polycyclic aromatic hydrocarbons and polychlorinated biphenyls in green-lipped mussel Perna viridis from Victoria Harbour, Hong Kong and possible human health risk, Marine Pollution Bulletin 58(4) (2009) 615-620. https://doi.org/10.1016/j.marpolbul.2008.12.016. 
[4] X.S. Zhao, J. Ding, H. You, Spatial distribution and temporal trends of polycyclic aromatic hydrocarbons (PAHs) in water and sediment from Songhua River, China. Environmental Geochemistry and Health 36(1) (2014) 131-143. https://doi.org/ 10.1007/s10653-013-9524-0.

[5] C.L. Ke, Y.G. Gu, Q. Liu, L.D Li, H.H Huang, N.Cai, and Z.W. Sun, Polycyclic aromatic hydrocarbons (PAHs) in wild marine organisms from South China Sea: Occurrence, sources, and human health implications. Marine Pollution Bulletin 117(1) (2017) 507-511. https://doi.org/10. 1016/j.marpolbul.2017.02.018.

[6] R.X. Sun, Q.Lin, C.L. Ke, F.Y. Du, Y.G. Gu, K. Cao, X.J. Luo, and B.X. Mai, Polycyclic aromatic hydrocarbons in surface sediments and marine organisms from the Daya Bay, South China. Marine Pollution Bulletin 103(1) (2016) 325-332. https://doi.org/10.1016/j.marpolbul.2016.01.009.

[7] Y.G. Gu, Q. Lin, H.H. Huang, L.G. Wang, J.J. Ning, F.Y. Du, Heavy metals in fish tissues/ stomach contents in four marine wild commercially valuable fish species from the western continental shelf of South China Sea. Marine Pollution Bulletin 114(2) (2017) 1125-1129. https://doi.org/10.1016/ j.marpolbul.2016.10.040.

[8] A. Shahbazi, M.P. Zakaria, C.K. Yap, S. Surif, A.R. Bakhtiari, K. Chandru, P.S. Bahry \& M. Sakari, Spatial distribution and sources of polycyclic aromatic hydrocarbons (PAHs) in green mussels (Perna viridis) from coastal areas of Peninsular Malaysia: implications for source identification of perylene, International Journal of Environmental Analytical Chemistry 90(1) (2010) 14-30. https://doi.org/10.1080/0306731090 2913000
[9] Y. Miguel-Gallo, M. Gómez-Batista \& C.M. Alonso-Hernández, Levels of Polycyclic Aromatic Hydrocarbons in Perna viridis, in Cienfuegos Bay, Cuba, Polycyclic Aromatic Compounds 39(2) (2017) 139-147. https://doi.org/10.1080/10406638. 2017.1293697.

[10] Z.L. Yu, Q. Lin, Y.G. Gu, C.L. Ke, and R.X. Sun, Spatial-temporal trend and health implications of polycyclic aromatic hydrocarbons (PAHs) in resident oysters, South China Sea: A case study of Eastern Guangdong coast. Marine Pollution Bulletin 110(1) (2016) 203-211. https://doi.org/10. 1016/j.marpolbul.2016.06.061.

[11] J. Shi, G.J.S. Zheng, M.H. Wong, H. Liang, Y. Li, Y. Wu, P. Li, W. Liu, Health risks of polycyclic aromatic hydrocarbons via fish consumption in Haimen bay (China), downstream of an e-waste recycling site (Guiyu). Environmental Research, 147 (2016) 233-240. https://doi.org/10.1016/j.envres. 2016.01.036.

[12] C. Ding, H.G. Ni, and H.Zeng, Human exposure to parent and halogenated polycyclic aromatic hydrocarbons via food consumption in Shenzhen, China. Science of The Total Environment 443 (2013) 857-863. https://doi.org/10.1016/j.scitotenv. 2012.11.018.

[13] E. Yoon, K. Park, H. Lee, J.H. Yang, C. Lee, Estimation of excess cancer risk on time-weighted lifetime average daily intake of PAHs from food ingestion. Human and Ecological Risk Assess 13 (2007) 669-80. https://doi.org/10.1080/10807030 701226871.

[14] R. Martí-Cid, J.M. Llobet, V. Castell, J.L. Domingo, Evolution of the dietary exposure to polycyclic aromatic hydrocarbons in Catalonia, Spain. Food and Chemical Toxicology 46 (2008) 3163-3167. https://doi.org/10.1016/j.fct.2008.07.002. 